\title{
Schistosoma mansoni TOR is a tetraspanning orphan receptor on the parasite surface
}

\author{
C. LOCHMATTER*, J. A. SCHIFFERLI and P. J. MARTIN \\ Department of Biomedicine, Immunonephrology, University Hospital Basel, Hebelstrasse 20, CH-4031 Basel, Switzerland
}

(Received 19 September 2008; 15 December 2008 and 18 Fanuary 2009; accepted 18 Fanuary 2009; first published online 13 March 2009)

\begin{abstract}
S U MMARY
A trispanning orphan receptor (TOR) has been described in Schistosoma haematobium and S. mansoni. Here we report the complete molecular organization of the $S$. mansoni TOR gene, also known as SmCRIT (complement C2 receptor inhibitor trispanning). The SmTOR gene consists of 4 exons and 3 introns as shown by cloning the single exons from $S$. mansoni genomic DNA and the corresponding cDNA from the larval stage (cercaria) and the adult worm. The SmTOR ORF consists of $1260 \mathrm{bp}$ and is longer than previously reported, with a fourth trans-membrane domain (proposed new name: Tetraspanning Orphan Receptor) and with, however, an unchanged C2-binding domain on the extracellular domain 1 (ed1). This domain differs in S. japonicum. A protein at the approximate expected molecular weight (55 kDa) was detected in adult worm extracts with polyclonal and monoclonal antibodies, and was found to be expressed on the tegumental surface of cercariae.
\end{abstract}

Key words: CRIT, TOR, Schistosoma, complement regulation.

\section{INTRODUCTION}

Schistosomiasis is a parasitic infection also known as bilharzia named after Theodor Bilharz who first described it (Ross et al. 2002). There are 5 species of schistosoma that are known to infect humans by contact with its larval stage, the cercariae: Schistosoma mekongi, S. intercalatum, S. mansoni, S. japonicum and $S$. haematobium, the latter 3 being the main schistosome species that affect humans (Hamburger et al. 1998). Adult worm pairs reside in host veins and produce eggs which penetrate the tissue. They end up excreted with feces where they complete the schistosoma life cycle by hatching and re-infecting fresh-water snails as intermediate hosts (Ross et al. 2002). Between the mid-1990s and 2003 the estimated number of people at risk from schistosomiasis increased from 702 million to 779 million and the estimated number of individuals infected increased from 193 million to 207 million (Steinmann et al. 2006). The treatment of choice is the chemotherapeutic agent praziquantel. However, drug treatment alone might not be sufficient and a vaccine-linked chemotherapy to control schisotomiasis is recommended (Bergquist et al. 2005). Naturally acquired immunity or vaccination in animals strongly diminishes the pathology associated with schistosome infection. There are efforts to develop a vaccine against schistosomiasis. Radio-attenuated cercariae induced

* Corresponding author. Tel: +4161 2653891. Fax: +41 61 2652350. E-mail: c.lochmatter@unibas.ch a high level of protection in animal models (Minard et al. 1978; Stek et al. 1981) and naturally resistant population groups exist (Correa-Oliveira et al. 1989, 2000), which suggests that development of an effective vaccine is likely to be possible (McManus and Loukas, 2008). There is a list of recombinant proteins that correlate with resistance in human studies and/or have shown efficacy in animal models (McManus and Loukas, 2008). Among these promising vaccine candidates are the tetraspanins, SmTSP-1 and SmTSP-2, that are both recognized by $\mathrm{IgG} 1$ and $\mathrm{IgG} 3$ from putatively resistant individuals, SmTSP-2 providing high level of protection in the mouse vaccination model in addition (Tran et al. 2006). Another candidate, Fatty Acid-Binding Protein (FABP)-Sm14 is at the stage of planned clinical trials after scale-up and industrial production processes have been put in place (Tendler and Simpson, 2008).

The host complement system participates in the first line of immune defences against the invading parasite. Of the 3 pathways of complement activation, the alternative pathway was shown to attack schistosomula (Ruppel et al. 1984; Pearce et al. 1990) and $S$. mansoni adult worms (Linder and Huldt, 1983; Rasmussen and Kemp, 1987). Schistosomes are sensitive to killing by complement, but lose their sensitivity with loss of the glycocalyx and maturation. Partial tryptic digestion of adult worm tegumental proteins rendered them sensitive to complement attack as shown for $S$. mansoni by Fishelson's group (Marikovsky et al. 1990). The 
mannose-binding lectin pathway has also been shown to be activated (Klabunde et al. 2000), but there has been no consensus regarding the activation of the classical pathway. Some researchers find $\mathrm{IgG}$ (different subtypes) and IgM deposits at the parasite surface and some do not (Kemp et al. 1976, 1978, 1980). Identification of host IgG1, IgG3, IgM, C3 degradation products (Braschi and Wilson, 2006) and C4 (Braschi et al. 2006 a), but no known proteins with homology to $\mathrm{Fc}$ receptors by proteomic analysis of the adult worm tegument, provide a strong argument for activation of the classical pathway (Braschi et al. 2006a). In addition, there have been reports of complement regulators being present on schistosomes, including $\mathrm{C} 1 \mathrm{q}$ binding proteins, surface $\mathrm{C} 3$ receptor, host acquired DAF and SCIP-1/paramyosin (Skelly, 2004), which might regulate the terminal membrane attack complex insertion, although this remains a matter of debate (Skelly and Wilson, 2006).

A specific surface receptor for C2, the Schistosoma trispanning orphan receptor (TOR) was described by Inal some time ago (Inal, 1999). In S. haematobium and S. mansoni TOR was found to be a $32 \mathrm{kDa}$ trans-membrane protein located at the tegumental surface of adult worms and was also expressed in the larval stage (cercaria). Inal showed that a short amino acid sequence of the ShTOR extracellular domain 1 (ed1) binds $\mathrm{C} 2$, resulting in competitive inhibition of the binding of $\mathrm{C} 2$ to $\mathrm{C} 4 \mathrm{~b}$. In addition it inhibits the cleavage of $\mathrm{C} 2$ by $\mathrm{C} 1 \mathrm{~s}$. Further experiments indicated that this sequence was a strong inhibitor of classical pathway activation. This sequence, corresponding to the $\mathrm{C}$-terminal 11 amino acids of ed1 (termed H17 peptide), has homologies with a specific sequence of the beta chain of $\mathrm{C} 4$, which may explain the competitive binding between $\mathrm{H} 17$ and $\mathrm{C} 4$ for $\mathrm{C} 2$ (Inal and Schifferli, 2001, 2002). Hui et al. (2005) defined more precisely the binding site of $\mathrm{H} 17$ to be the vWFA domain of C2. Therefore, TOR was renamed CRIT for 'complement $\mathrm{C} 2$ receptor inhibitor trispanning' (Inal and Sim, 2000; Inal et al. 2005 b). Recently, H17 was shown to interfere also with the formation of the alternative pathway $\mathrm{C} 3$ convertase by binding to FB (Hui et al. 2005, 2006). Evidently, TOR might be a central element for schistosomes to escape innate immunity.

Here we report the exon/intron structure of the SmTOR gene. Four exons were amplified and sequenced from S. mansoni genomic DNA based on database analysis of the $S$. mansoni genome and comparing it with S. japonicum TOR (SjTOR) cDNA. The full-length construct was amplified from the adult worm and cercarial cDNA preparations. Further evidence for expression of the TOR protein was gained by Western blotting with $S$. mansoni proteins and probing these with antibodies directed against the extracellular domain. Based on these results we propose a new structure of SmTOR/CRIT.
MATERIALS AND METHODS

Alignment of $\mathrm{S}$. japonicum $c D N A$ with $\mathrm{S}$. mansoni genome : derivation of SmTOR exon/intron gene organization

SjTOR cDNA (PubMed Accession number AY814912; http://www.ncbi.nlm.nih.gov/) was aligned with S. mansoni GeneDB database entry Smp_ 093840 (http://www.genedb.org/genedb/smansoni/) designated as a putative trispanning orphan receptor gene. The resulting overlapping sequences served as a basis to define SmTOR exon/intron boundaries.

\section{PCR amplification and sequencing of SmTOR fragments from $\mathrm{S}$. mansoni genomic $D N A$}

S. mansoni genomic DNA was prepared from cercariae-infected water. Cercariae in suspension were washed by pelleting for $5 \mathrm{~min}$ at $1800 \mathrm{~g}$ and resuspending in $1 \times \mathrm{PBS}$ and an additional centrifugation step as before. The pellet was resuspended in $1 \times$ TE (Fluka 86377)/100 $\mathrm{mm} \mathrm{NaCl}$ and snap frozen in liquid nitrogen. After thawing, 20\% SDS (Fluka 05030) and Proteinase K (Fluka 82456) were added to a final concentration of $1 \%$ and $1 \mu \mathrm{g} / \mathrm{ml}$ respectively. The mixture was incubated at $60{ }^{\circ} \mathrm{C}$ overnight. One volume of TE saturated phenol/ chloroform was added to the sample and mixed by inversion for $15 \mathrm{~min}$. After spinning at full speed in a microfuge, the aqueous supernatant was transferred to a new tube and the extraction step repeated twice, but using chloroform only in the last cycle. DNA in the aqueous phase was then precipitated by adding $1 / 10(\mathrm{v} / \mathrm{v}) \mathrm{NaOAc}, \mathrm{pH} 5 \cdot 5$, overlaid with $2 \cdot 5$ volumes of ethanol and incubating at $-20{ }^{\circ} \mathrm{C}$ overnight. DNA was pelleted at full speed, air-dried and resuspended in $1 \times$ TE. Primers (Microsynth) flanking the putative SmTOR exons were designed according to the exon/intron map (Table 1). PCR was performed with Taq PCR core kit (Quiagen) using 0.75 mM specific primers and $30 \mathrm{ng}$ S. mansoni genomic DNA as template. The PCR programme was $95^{\circ} \mathrm{C}$ for $5 \mathrm{~min}$, then 35 cycles of $95{ }^{\circ} \mathrm{C}$ for $45 \mathrm{~s}, 59^{\circ} \mathrm{C}$ for $1 \mathrm{~min}$, $72{ }^{\circ} \mathrm{C}$ for $1 \mathrm{~min}$ followed by a final extension step of $72{ }^{\circ} \mathrm{C}$ for $15 \mathrm{~min}$. Reaction products were separated by $1 \%$ agarose gel electrophoresis, excised bands were purified (QIAquick ${ }^{\circledR}$ PCR purification kit) and cloned into a TOPO vector (Invitrogen) for sequencing. Blanks for each PCR reaction using water only were negative (not shown). All PCR products were sequenced in both directions, using plasmid preparations from 3 different clones respectively.

\section{RNA isolation from $\mathrm{S}$. mansoni adult worms or cercariae and full length $c D N A$ preparation}

S. mansoni RNA was isolated from adult worm preparations of $S$. mansoni (Liberian strain, kindly donated by Dr J. Chollet, STI, Basel) isolated from 
Table 1. Primer list used for amplification of single exons of SmTOR and amplifications from cDNA

(Expected fragment lengths are indicated for primer pairs used to amplify single exons. Numbers used in the primer names indicate its $5^{\prime}$ annealing position within the exon or the flanking intron, preceded by a positive or negative sign respectively, if not annealing at the ends of the exons of interest due to issues of melting temperature when designing.)

\begin{tabular}{lll}
\hline \hline Primer & Sequence 5' $\rightarrow 3^{\prime}$ & $\begin{array}{l}\text { Expected } \\
\text { product (bp) }\end{array}$ \\
\hline SmTOR_ex1_-15_fwd & GTCTCGTTAACTGTCGTTGTTGAATAATTG & $246 \mathrm{bp}$ \\
SmTOR_ex1_+12_rev & TCTTGTCCTCTGATGGGTCTGTATTTCCAT & $246 \mathrm{bp}$ \\
SmTOR_ex2_-12_fwd & TTCTACCCTAGGTTTTTATGTTTTCTCGAC & $538 \mathrm{bp}$ \\
SmTOR_ex2_rev & TTTTGTGTGAATCATCAAGCGTAGATCTGA & $538 \mathrm{bp}$ \\
SmTOR_ex3_fwd & ACGGGGCCTATTTACATCAAATCTACA & $325 \mathrm{bp}$ \\
SmTOR_ex3_rev & CTCATACTTTGGTAGATCGTTAGCTGG & $325 \mathrm{bp}$ \\
SmTOR_exon4_7_fwd & TATTGAAATTCCGGCAATTGCCTACGCTC & $248 \mathrm{bp}$ \\
SmTOR_exon4_rev & TTAGCAAGAAGAGTGAGCATTCGATGGTGC & $248 \mathrm{bp}$ \\
SmTPI_fwd & GTTGGGGGGAACTGGAAATGAA & $219 \mathrm{bp}$ \\
SmTPI_rev & TTCTCCGGTGATGCACCCTTTG & $219 \mathrm{bp}$ \\
SmTOR_ex1_-15_fwd & GTCTCGTTAACTGTCGTTGTTGAATAATTG & $291 \mathrm{bp}$ \\
SmTOR_ex2_+33_rev & ACAAGACGAAAGAGAGTCGAGAAAATATAA & $291 \mathrm{bp}$ \\
\hline \hline
\end{tabular}

NMRI mice or cercariae. Worm pairs were briefly rinsed with $1 \times$ PBS and preserved in RNAlater ${ }^{\circledR}$ reagent (Ambion). Worm tissue or cercariae were homogenized by mechanical disruption with Molecular Grinding Resin ${ }^{\mathrm{TM}}$ (G-Biosciences) resuspended in lysis buffer RLT (AllPrep ${ }^{\mathrm{TM}}$ DNA/RNA/ Protein extraction kit, Quiagen). After removal of resin and cell debris by centrifugation at $10800 \mathrm{~g}$, for $5 \mathrm{~min}$ at $4{ }^{\circ} \mathrm{C}$, the homogenate was applied on a Quiagen AllPrep column and RNA extracted according to the manufacturer's protocol.

\section{$R T-P C R$ and $c D N A$ alignment SmTOR/SjTOR}

Single strand cDNA synthesis was performed using random hexamers, oligo dT primers or gene specific primer SmTOR_exon4_rev (Table 1). Fifty ng of RNA was reverse transcribed per reaction with SuperScript ${ }^{\text {TM }}$ III First-Strand Synthesis System for RT-PCR (Invitrogen). Using the transcribed single stranded cDNAs as a template, the SmTOR transcribed sequence was amplified with gene specific primer pair SmTOR_ex1_-15_fwd and SmTOR_exon4_rev (Table 1) using the same cycling conditions as described above. Reaction mixtures were run on a $1 \%$ agarose gel and the single bands purified, cloned and analysed as described above. SmTOR ORF on sequenced cDNA was aligned with SjTOR ORF on cDNA entry (AY814912) using EMBOSS pairwise alignment algorithm (http:// www.ebi.ac.uk/emboss/align/).

\section{Real-time quantitative PCR analysis ( $P P C R)$}

Total RNA was isolated from S. mansoni cercaria, schistosomula, adult worm pairs, eggs and miracidia as described above. Eggs and miracidia were isolated as reported previously (Dalton et al. 1997). Schistosomula were generated by in vitro transformation
(Ramalhopinto et al. 1974). cDNA was generated as described above and $1 \mu \mathrm{l}$ per reaction was used, performing qPCR on an ABI 7900 (Applied Biosystems) instrument using $\mathrm{SYBR}{ }^{\circledR}$ green as a fluorescence dye (Power SYBR ${ }^{\circledR}$ Green PCR Master Mix, Applied Biosystems). Primers targeting a $219 \mathrm{bp}$ region of the constitutively expressed SmTPI (triose phosphate isomerase) (Hooker and Brindley, 1996) and primers used for SmTOR (SmTOR_ex1_-15_ fwd and SmTOR_ex2_+33_rev) are listed in Table 1. Amplification of contaminating genomic DNA was avoided by using sets of primers located in different exons. Results were evaluated using the $2^{-\Delta \Delta C_{T}}$ method (Livak and Schmittgen, 2001).

Protein sequence alignment of $\mathrm{S}$. japonicum and $\mathrm{S}$. mansoni TOR and secondary structure prediction of $\operatorname{SmTOR}$

Molecular weight analysis was performed using the EMBOSS Pepstats program at the European Bioinformatics Institute web site (http://www.ebi. ac.uk/emboss/pepinfo). Proteins were aligned using EMBOSS pairwise alignment algorithm (http:// www.ebi.ac.uk/emboss/align/). TopPred was used for transmembrane prediction analysis (Claros and Vonheijne, 1994) using upper cutoff hydrophobicity values. Secondary structure prediction analysis was performed using PSIPREDView (Jones, 1999; McGuffin et al. 2000). The signal sequence prediction was performed using SignalP 3.0 server (http:// cbs.dtu.dk/services/SignalP).

\section{Adult worm tegument membrane preparations and Western blot analysis}

Tegument surface membranes of $S$. mansoni adult worms were prepared as done previously, applying 
the freeze-thaw method followed by vortex pulses in order to strip the parasites (Roberts et al. 1983; Brouwers et al. 1999). Samples were run on a standard 12\% SDS-PAGE acrylamide/bisacrylamide $(30 \% / 0 \cdot 8 \%$, Bio-Rad) gel and proteins then transferred to a nitrocellulose membrane (162-0115, BioRad). After blotting, the membrane was stained with Ponceau red. Subsequently, the membrane was blocked in $1 \times$ PBS $/ 0 \cdot 05 \%$ Tween 20 (Sigma) $/ 5 \%$ milk (170-6404 Bio-Rad) for $1 \mathrm{~h}$ at RT. Incubations with primary and secondary antibodies described below were done in $1 \times \mathrm{PBS} /$ Tween $0.05 \% / 1 \%$ blotting milk (Bio-Rad) with 3 washing steps of 5 min shaking at RT before developing the blot. Two polyclonal antibodies generated respectively against SmTOR peptide ed1 $\mathrm{NH}_{2}$-MSPSLVSYTQKNERGSHEVKIKHFSP-COOH (Inal and Schifferli, 2002) and against ShCRIT peptide ed2 $\mathrm{NH}_{2}$-SSTSDIRLVIHTKTGPIYIKST-COOH 1:1000 were used in PBS/T 1:1000 (Inal et al. 2005b), followed by goat anti-rabbit IgG-HRP coupled (Bio-Rad, \#1706515). The blot was developed using the ECL TM Western blotting detection system (Amersham Biosciences). Alternatively, a human monoclonal antibody against SmTOR peptide ed1 isolated from a human monoclonal antibody library (HuCAL) was used at a dilution of $1: 1000(2.52 \mathrm{mg} / \mathrm{ml}$ stock $)$ in PBS/T (AbyD04644.1, AbD Serotec, Martinsried). Mouse anti-Histidine-tag:HRP (MCA1396P, Serotec) $1: 3000$ in PBS/T was used as secondary antibody and the blot revealed as described above. The antibody was blocked by pre-incubation of AbyD0644.1 with a 100-fold molar excess of ed1 peptide in $\mathrm{PBS} / \mathrm{T}$ for $60 \mathrm{~min}$ at RT, and subsequent steps were performed as described above.

\section{Electron microscopy}

Cercariae were fixed in 3\% paraformaldehyde and $0.5 \%$ glutaraldehyde in $10 \mathrm{~mm}$ PBS $(\mathrm{pH} \mathrm{7 \cdot 4})$. After washing with $\mathrm{PBS}$, they were treated with $0 \cdot 5 \% \mathrm{OsO}_{4}$ for $30 \mathrm{~min}$ followed by dehydration in a series of graded ethanol solutions and embedding in LR White resin at $60{ }^{\circ} \mathrm{C}$. Ultrathin sections $(60 \mathrm{~nm})$ were cut on an UltracutE Leica ulramicrotome and collected on copper 200 mesh grids. Grids were blocked in PBS $/ 2 \%$ BSA for $2 \times 5 \mathrm{~min}$. Polyclonal anti-ed 1 or anti-ed 2 antibodies described before were diluted in blocking buffer $(1: 50)$ and pre-immune serum was used as control. After incubation with primary antibodies for $2 \mathrm{~h}$, grids were washed twice for $5 \mathrm{~min}$ with blocking buffer and incubated for $1 \mathrm{~h}$ with goat anti-rabbit IgG (EM-GAHL10, British BioCell Laboratories) diluted 1:20 in blocking buffer. Final washings were performed followed by staining with $6 \%$ uranyl acetate, for $1 \mathrm{~h}$, and then Millonigs lead acetate for $2 \mathrm{~min}$. The sections were then dried, examined and photographed using a Philips Morgani transmission electron microscope.

\section{Cryosections and immunolocalization}

Thin sections $(9 \mu \mathrm{m})$ of OCT-embedded frozen $S$. mansoni cercariae were cut on a cryostat (Microm HM 560) and fixed in ice-cold methanol for $10 \mathrm{~min}$. After blocking in $1 \times \mathrm{PBS} / 3 \% \mathrm{BSA}$ sections were stained with monoclonal antibodies against ed1 (AbyD04644.1, described above) $1: 50$ in $1 \times \mathrm{PBS} /$ $1 \%$ BSA or anti-GFP antibody (AbyD04652, AbD Serotec, Martinsried, $1.14 \mathrm{mg} / \mathrm{ml}$ stock) diluted to the according concentration for $2 \cdot 5 \mathrm{~h}$ at RT. A fluorescein-labelled goat anti-human $\operatorname{IgG} \mathrm{F}\left(\mathrm{ab}^{\prime}\right)_{2}$ specific secondary antibody (\# 109-095-006, Jackson Immuno Research Laboratories) was used at a dilution of $1: 100$ in PBS/BSA for $30 \mathrm{~min}$ at RT. Slides were mounted with Vectashield fluorescence mounting medium (Vector Laboratories) and examined using LSM 510 META confocal laser scanning microscopy system (Carl Zeiss, Feldbach, Switzerland) with a Zeiss Plan Neofluar 63x/1.25 numeric aperture oil $(\infty / 0 \cdot 17)$ objective.

\section{RESULTS}

\section{S. mansoni TOR gene comprises four exons}

Thanks to $S$. mansoni genome sequence data published on the GeneDB website, we performed an alignment with $S$. japonicum mRNA sequence available in the NCBI database in order to define exon/intron boundaries of the SmTOR gene. The analysis suggested that the SmTOR gene comprised 4 exons, instead of the 3 suggested in GeneDB. Figure 1 represents the exon localizations along the Sm genome, showing that the gene contains 3 introns in the range of 2500 to $3500 \mathrm{bp}$, resulting in a $9721 \mathrm{bp}$ length gene. The hatched boxes in $\mathrm{Sj}$ mRNA represent the $5^{\prime}$ and $3^{\prime}$ untranslated regions. The resulting transcript and protein would thus be longer than observed before (Inal, 1999). Thanks to this alignment, we were able to locate the ATG start codon of the SmTOR gene Smp_093840 at position 60992 in scaffold Smp_scaff000547.

\section{Cloning and sequencing of SmTOR exons}

We cloned the single exons from genomic DNA extracted from S. mansoni cercariae using primers flanking the exons (Table 1). Separation of the PCR products by agarose gel electrophoresis showed bands at the expected size (Fig. 2A). The product for the amplification of exon 2 was expected to be $538 \mathrm{bp}$ long but appears to be about $50 \mathrm{bp}$ smaller. After gel extraction, cloning and sequencing of the single bands, the PCR product of exon 2 was found to be $481 \mathrm{bp}$ long. The difference of $50 \mathrm{bp}$ is because part of this region in the $S$. mansoni GeneDB is unknown and designated as multiple NNN. The length and sequencing results of the other $3 \mathrm{PCR}$ products did match the information deposited at the S. mansoni 


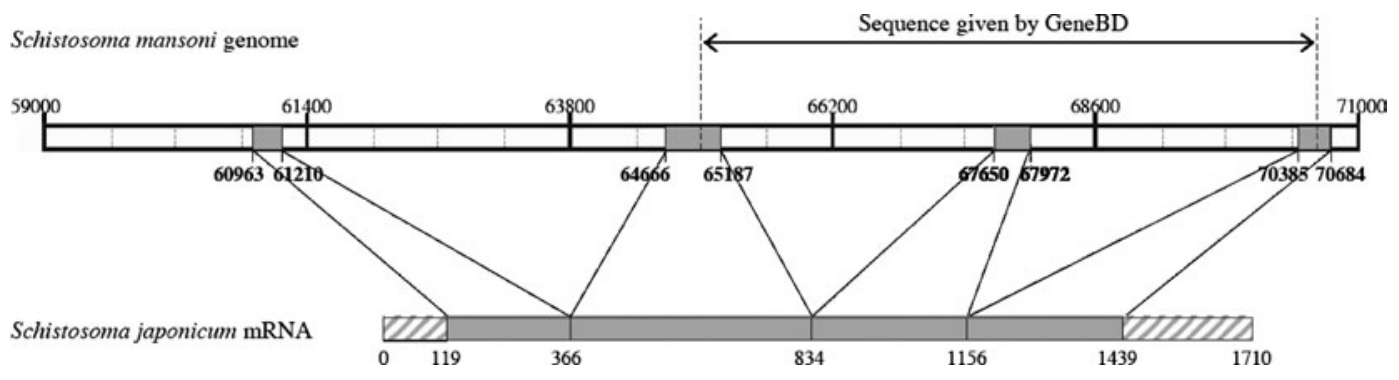

Fig. 1. Alignment of Sm (Schistosoma mansoni) genome (Smp_093840) and Sj (Schistosoma japonicum) mRNA of CRIT. Representation of the gene structure of SmCRIT exons and introns. The grey boxes represent the exons, the hatched boxes represent the sequences not found in Sm genome. Numbers indicate positions on scaffold Smp_scaff000547.

A

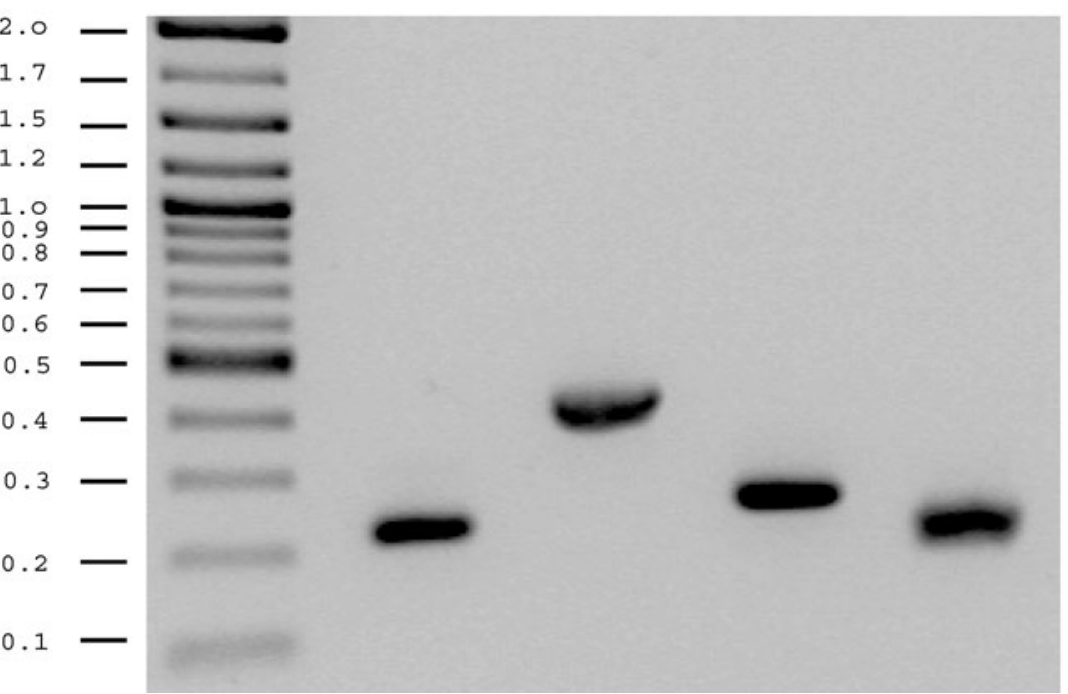

B

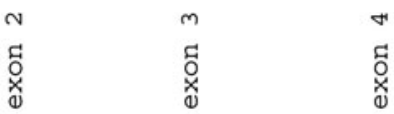

돈 $\mathbf{B}$
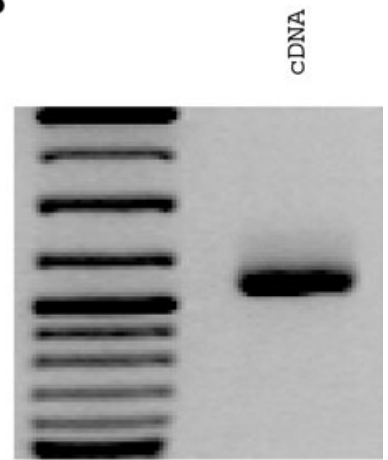

Fig. 2. Electrophoretic separation of PCR amplification products from Schistosoma mansoni genomic DNA or adult worm cDNA. (A) Lanes 1-4 correspond to PCR amplification of exon 1-4 respectively, primers used Table 1.

(B) SmTOR ORF amplified from adult worm cDNA, primers: smTOR_ex1_-15_fwd, smTOR_exon4_rev (Table 1).

GeneDB database. All the fragments were cloned at least 3 times and sequenced in both directions. Sequencing results of the single exons are in complete concordance with the cDNA sequence shown below in an alignment with SjTOR cDNA (Fig. 3). This delineation of sequencing information represents the merged information of single exon sequencing results. How the gained information is related to the GeneDB entry is listed in the next section.

\section{Expression of full-length SmTOR}

PCR amplification of SmTOR ORF from cDNA preparations from $S$. mansoni adult worms and cercariae generated with different primers (as described above) yielded the same result for all the reactions. Size-separation of reaction products amplified with smTOR_ex1_-15_fwd and smTOR_exon4_rev showed a single band at the expected size (Fig. 2B).
The gel-purified and cloned fragments all held the $1260 \mathrm{bp}$ sequence coding for full length SmTOR. The results were identical using different clones and performing sequencing reactions. The sequencing result for SmTOR cDNA is shown in alignment with SjTOR cDNA (AY814912) starting at the translation initiation codon (Fig. 3). When aligning the results from the single exons described in the previous section with the sequenced SmTOR cDNA the correlation was $100 \%$. Splicing sites are marked with black arrows on the SmTOR cDNA (Fig. 3).

Sequencing data for SmTOR cDNA perfectly match the corresponding sequences in the $S$. mansoni GeneDB database (positions 60991 to 70636, scaffold Smp_scaff000547) on S. mansoni genome (starting at ATG on exon 1) for exons 1, 3 and 4 (single mutations differing between the clones and occuring at a frequency of 2 per $1260 \mathrm{bp}$ were not taken into account). The gap in the $S$. mansoni GeneDB database lying within SmTOR exon 2 


\begin{tabular}{|c|c|c|c|c|}
\hline sjTOR & CDNA & $\begin{array}{l}1 \\
1\end{array}$ & 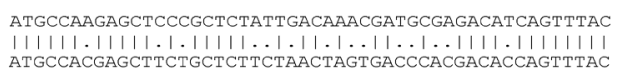 & 50 \\
\hline SMTOR & CDNA & 51 & $\begin{array}{l}\text { GTGCTGCCTTTGCTTACATGTGAGGACAGGGACGATAATTTTCGGGATAA } \\
\text {. }\end{array}$ & 100 \\
\hline sjTOR & CDNA & 51 & CTGTTGCCTTTGTCTACATGTGAGGACAGGGACTATAATCTTCGGAATAA & 100 \\
\hline SMTOR & CDNA & 101 & $\begin{array}{l}\text { CCCAGATAATATCCAACTTGTCTIATATCCTTITATTCCTGATGACG } \\
|\cdot| l|l| l|l| l|l| l|l| l|l| l|l| l|l| l|l| l|l| l|l| l|l| l\end{array}$ & 150 \\
\hline SJTOR & CDNA & 101 & CTCAAATTATTATCCAACTCATCT TIATATCGTTTCTTTTCCTAATGACG & 150 \\
\hline SMTOR & CDNA & 151 & 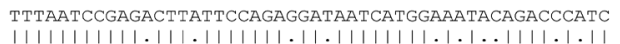 & 200 \\
\hline SJTOR & CDNA & 151 & TTTAATCCGAGGCTTTTTCCAGAAGACAATCATGGGAGTTTAGACTCCTC & 200 \\
\hline SMTOR & CDNA & 201 & 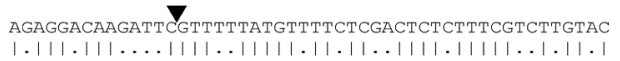 & 250 \\
\hline SJTOR & CDNA & 201 & ACAGGCCAACGCCCGTTACTATGTCTTATCAGCTCTGTTTCGCTTAGTGC & 250 \\
\hline SMTOR & CDNA & 251 & 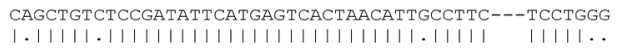 & 297 \\
\hline sJTOR & CDNA & 251 & CTGCTGTITCCGATATTCATGAGTCACTAACATTTCCTICCTTICCTGAA & 300 \\
\hline SMTOR & CDNA & 298 & 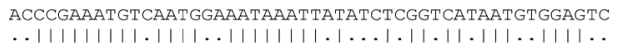 & 347 \\
\hline SJTOR & CDNA & 301 & GTCCGAAATGTTAATGACAATAAATTGTTATTTGGCCACAATTCGGAGAG & 350 \\
\hline SMTOR & CDNA & 348 & 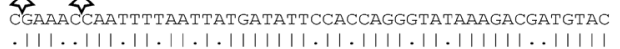 & 397 \\
\hline SJTOR & CDNA & 351 & TGAAGTCAACTTCAACTTTGATATTTCATCAGGTTACAAAGACAGTGTAC & 400 \\
\hline SMTOR & CDNA & 398 & 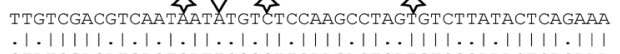 & 447 \\
\hline SJTOR & CDNA & 401 & CTATCGACATGAGTCATTCGCCTTCAAGACTCATGTCCGAAACTCACAAA & 450 \\
\hline SMTOR & CDNA & 448 & 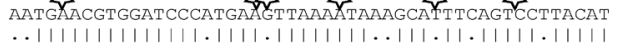 & 497 \\
\hline sjTOR & CDNA & 451 & CGTGAACGTGGATCCCGTGAAATTAAAATACGGCAGTTTAGTCCATACAT & 500 \\
\hline smTOR & CDNA & 498 & 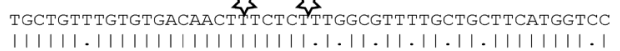 & 547 \\
\hline SJTOR & CDNA & 501 & TGCTGTCTGTGTGACAACTTTCTCTCTTGCTTTCTGTTGTTTCATGGTTC & 550 \\
\hline SMTOR & CDNA & 548 & 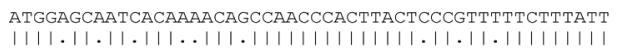 & 597 \\
\hline sjTOR & CDNA & 551 & ATGGGGCTATTACACGACAACCAACCCACTTACTTCCATTCT TCTTTATT & 500 \\
\hline SMTOR & CDNA & 598 & 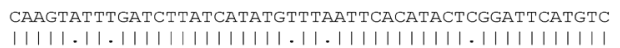 & 647 \\
\hline SJTOR & CDNA & 601 & CAAGTCTICGATCTTATCATATGCTTGATTCACATACTTGGATTCATGTC & 650 \\
\hline SMTOR & CDNA & 648 & 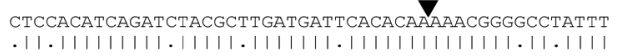 & 697 \\
\hline sJTOR & CDNA & 651 & TTCAACATCAGATATACGCCTGATGATCCACACAAAAACGGGACCAATTT & 700 \\
\hline SMTOR & CDNA & 698 & 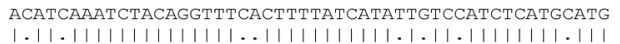 & 747 \\
\hline sJTOR & CDNA & 701 & ATATTAAATCTACAGGTTTAGCTTTTATCATACTATCTATCTCATGTATG & 750 \\
\hline SMTOR & CDNA & 748 & $\begin{array}{l}\text { ATGTTGGCTTTCAAAGCCTATTGTCTTGGTATGGTATGGGACTGTTATAA } \\
\text { ll }\end{array}$ & 797 \\
\hline SJTOR & CDNA & 751 & ATGTTGGCTTTCAAAGCCTACTGTCTTGGTATGGTGTGGGATTGTTATAA. & 800 \\
\hline SMTOR & CDNA & 798 & 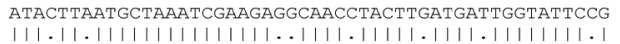 & 847 \\
\hline SJTOR & CDNA & 801 & ATATTTGATGCTAAATCGAAGAAACAACGTACTTAATGAGTGGTATTCTG & 850 \\
\hline SMTOR & CDNA & 848 & 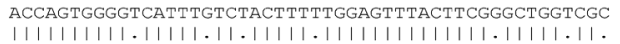 & 897 \\
\hline sjTOR & CDNA & 851 & ACCAGTGGGGCCATTTCTCAACTTTCTGGAGTTTACTTCGTGCTGGACGT & 900 \\
\hline SMTOR & CDNA & 898 & 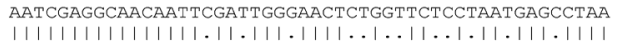 & 947 \\
\hline SJTOR & CDNA & 901 & AATCGAGGCAACAATTTGACTGGTAACTTAGACTCAGCCAACGAGTCTAA & 950 \\
\hline SMTOR & CDNA & 948 & 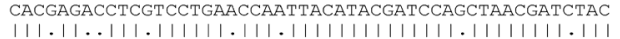 & 997 \\
\hline SJTOR & CDNA & 951 & CACAAGGGCTCATCCTGATCCAGTTACATACGATCCATCTAACGATTTAC & 1000 \\
\hline SMTOR & CDNA & 998 & 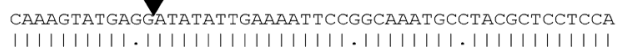 & 1047 \\
\hline sjTOR & CDNA & 1001 & CAAAGTATGATGATATATTGAAAATTCCAGCAAATGCT TACGCTCCTCCA & 1050 \\
\hline smTOR & $\mathrm{CDNA}$ & 1048 & 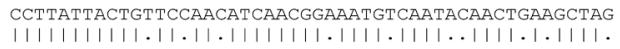 & 1097 \\
\hline sjTOR & CDNA & 1051 & ССТTATTACTGCTCTAATATCAACGGGAATGGCAATTTAACTCAGGCTAA & 1100 \\
\hline smTOR & $\mathrm{CDNA}$ & 1098 & 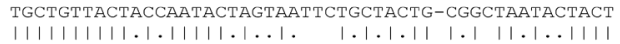 & 1146 \\
\hline sjTOR & CDNA & 1101 & TGCTGTTACTGCTAATACAACAAG---TACGAATGTCAG-TACTTTTACT & 1146 \\
\hline smTo & CDNA & 1147 & 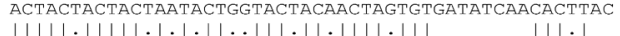 & 1196 \\
\hline SJTOR & CDNA & 1147 & 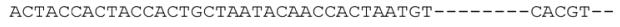 & $118 \mathrm{E}$ \\
\hline SMTOR & $C D N A$ & 1197 & 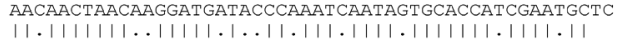 & 1246 \\
\hline sjTOR & CDNA & 1187 & --CAGCTAACAAAAATGATGCTGAAGTCACTAGTACACCATCAAATGTTC & 1234 \\
\hline smTo & $\mathrm{CDN}$ & 1247 & $\begin{array}{l}\text { ACTCTTCTTGCTAA } \\
|1||1| 1|1|\end{array}$ & \\
\hline SJTOR & & 1235 & A-TC--CTTGCTAA & \\
\hline
\end{tabular}

Fig. 3. SmTOR and SjTOR cDNA alignment; identical nucleotides are linked with a bar. Filled triangles indicate the positions of splice site on SmTOR cDNA (positions 214/215, 684/685 and 1009/1010, numbering with respect to the SmTOR start codon). Stars indicate mutations or gaps comparing sequencing data and GeneDB entry data; ATG of smTOR $0.86 \mathrm{~kb}$ ORF (AF051138) is indicated by a triangle. Bases 368-400 were newly sequenced on Schistosoma mansoni cDNA and from genomic DNA as described.

(position 368-400 on SmTOR cDNA, 64816 to 64 908, Smp_scaff000547 on S. mansoni GeneDB entry Smp_093840) could be filled in (Fig. 3).
Furthermore, we found 13 single nucleotide differences or gaps (indicated with stars in Fig. 3) within exon 2 in the range of position 348 to 400 when 


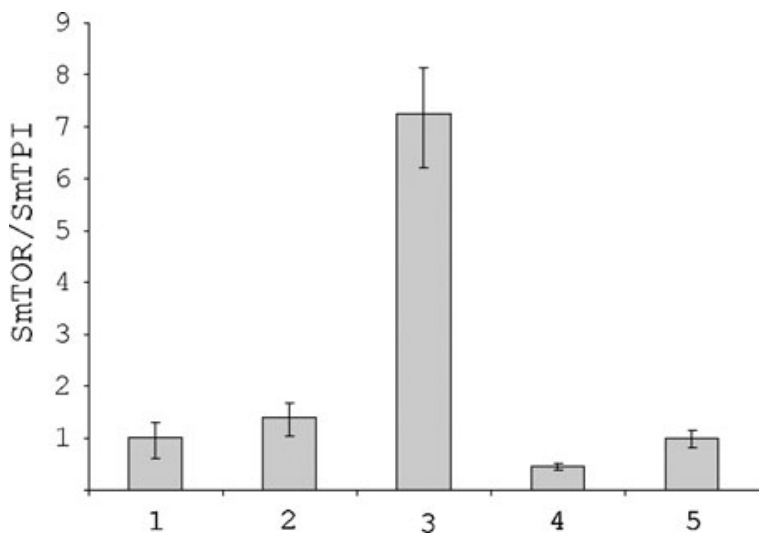

Fig. 4. Quantitative RT-PCR analysis of SmTOR mRNA in different Schistosoma mansoni stages: (1) eggs, (2) miracidiae, (3) cercariae, (4) schistosomulae, (5) adult worm. Expression of SmTOR quantitated relative to SmTPI as the control gene with bars representing $2^{-\Delta \Delta C_{T}}$ values.

comparing S. mansoni GeneDB sequence and SmTOR cDNA. The newly sequenced SmTOR cDNA compared with the shorter SmTOR mRNA database entry (AF051138) of Inal (1999) was nearly identical (18 single bases differed; the ATG is marked with a opened arrow (Fig. 3)). The former 5' UTR region published at the same time for $S$. haematobium TOR (64 base pairs upstream of the former ATG) matches the sequence we now find to be part of exon 2 of the longer SmTOR version, with only 5 bases being altered (not shown).

Developmental expression profiling of SmTOR as compared to SmTPI showed that the receptor mRNA was expressed at all the stages examined (Fig. 4). The receptor was expressed at a higher level in cercariae as compared to schistosomula, adult worm, egg and miracidia.

The cDNA sequences aligned for $S$. mansoni and S. japonicum TOR showed $46 \%$ homology at the nucleotide level.

\section{SmTOR translates into a protein similar in length to SjTOR and possesses four transmembrane domains}

SmTOR cDNA contains a $1260 \mathrm{bp}$ open reading frame which should generate a protein of 419 amino acid length with a theoretical mass of $46 \cdot 7 \mathrm{kDa}$. Its amino acid sequence is shown in alignment with SjTOR, a 414 amino acid protein based on proteomics data (Liu et al. 2006). Protein alignment of a possible SmTOR transcript and SjTOR shows $76 \cdot 7 \%$ homology on the protein level (Fig. 5).

Based on results from transmembrane prediction analysis we suggest SmTOR to have 4 transmembrane segments and a long C-terminal tail in homology with SjTOR (Fig. 6). In the absence of an $\mathrm{N}$-terminal signal peptide, the transmembrane domain 1 (aa 31-53, Fig. 6) is most likely to function as a signal anchor sequence for ER targeting and membrane insertion, as predicted with the SignalP software. The 4 transmembrane segments have an alpha helical conformation predicted with high confidence, and the amphipatic alpha helix in the intracellular domain 2 is likely to be partially inserted into the plasma membrane. The domain organization is the same for SmTOR and SjTOR. The overall transmembrane architecture remains the same as compared to the truncated protein version (UniProtKB/ TrEMBL entry Q9U597) (Inal et al. $2005 a, 2006$ ).

The 11 amino acids in the C-terminal part of ed1 that have been described to bind to $\mathrm{C} 2$ and interfere with its cleavage are boxed (Fig. 6). This sequence is different in SmTOR and SjTOR.

Performing Western blot analysis of adult worm tegument preparations we detected a protein of about $55 \mathrm{kDa}$ using antibodies generated against peptide sequences of ed1 and ed2 (Fig. 7). The same result was obtained using a monoclonal antibody generated against ed1 (Fig. 8A). Staining of cryosections of cercariae with the monoclonal antibody generated against ed1 showed surface labelling, as compared to the negative control (Fig. 8B). The presence of SmTOR on the tegument surface was confirmed by electron microscopy. In sections stained with the polyclonal antibodies against SmTOR ed1 and ed2 respectively, immunogold labelling was detected at the tegument surface (Fig. 9).

\section{DISCUSSION}

The data presented here clarify the exon/intron gene structure of SmTOR and provide evidence that $S$. mansoni expresses the corresponding protein.

We found that SmTOR gene structure contains 4 exons. A transcript containing the $1.26 \mathrm{~kb}$ SmTOR ORF showing $46 \%$ identity with the $1 \cdot 25 \mathrm{~kb}$ SjTOR ORF was found in adult worm and cercariae cDNA preparations. The sequencing data generated not only complete the gaps present in the $S$. mansoni GeneDB for SmTOR, but also correct some likely sequencing errors present in the database entry (stars on SmTOR cDNA Fig. 3). The sequencing data are likely to be correct. SmTOR ORF presented here aligns with the previously described SmTOR ORF on mRNA entry AF0511378 from position 418 on (alignment not shown), and translates into a functional protein aligning with $\mathrm{Sj}$ TOR. SmTOR is expressed at various developmental stages of S. mansoni. The highest expression was in cercariae, where we were able to detect it at the surface by confocal microscopy of cryosections and by electron microscopy. The higher expression in cercariae as compared to adult worms has previously been described for the truncated receptor version (Inal, 1999). This observation is of interest since cercariae are the first to come into contact with the human skin, an encounter that might determine the fate of the 


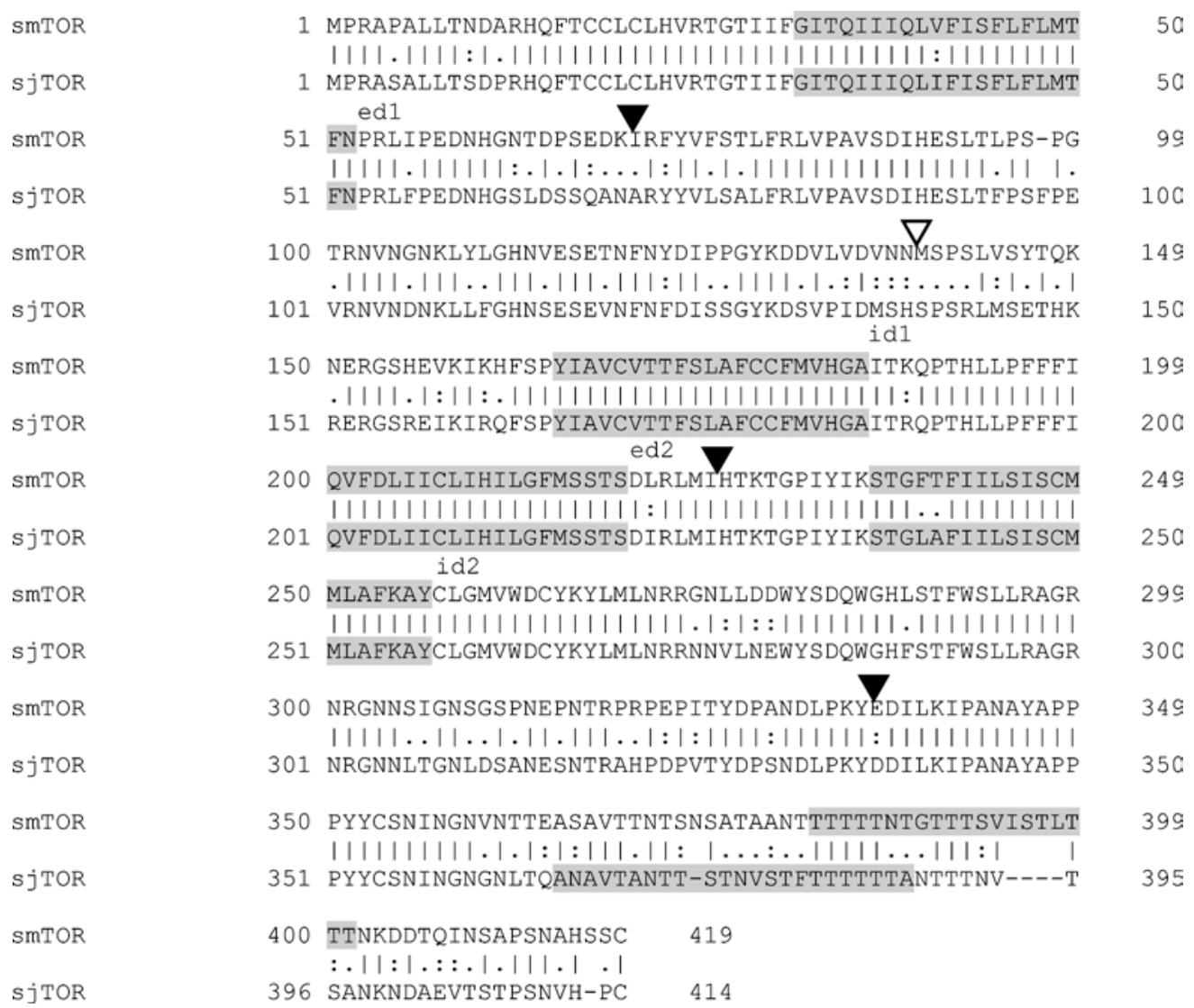

Fig. 5. SjTOR and SmTOR protein sequence alignment. Identical amino acids are linked with a bar (76.7\% identity), similar amino acids are connected via ' .' (84\% similarity). Exon/Intron boundaries on cDNA level for SmTOR are indicated with filled triangles. SmTOR protein (AF051138) starting with former extracellular domain 1 is marked by a triangle. Transmembrane domains 1-4 are shaded in grey as well as partially inserted helix 5. Domain designation: ed1, new extracellular domain 1; ed2, extracellular domain 2; id1, id2 intracellular domains 1 and 2.

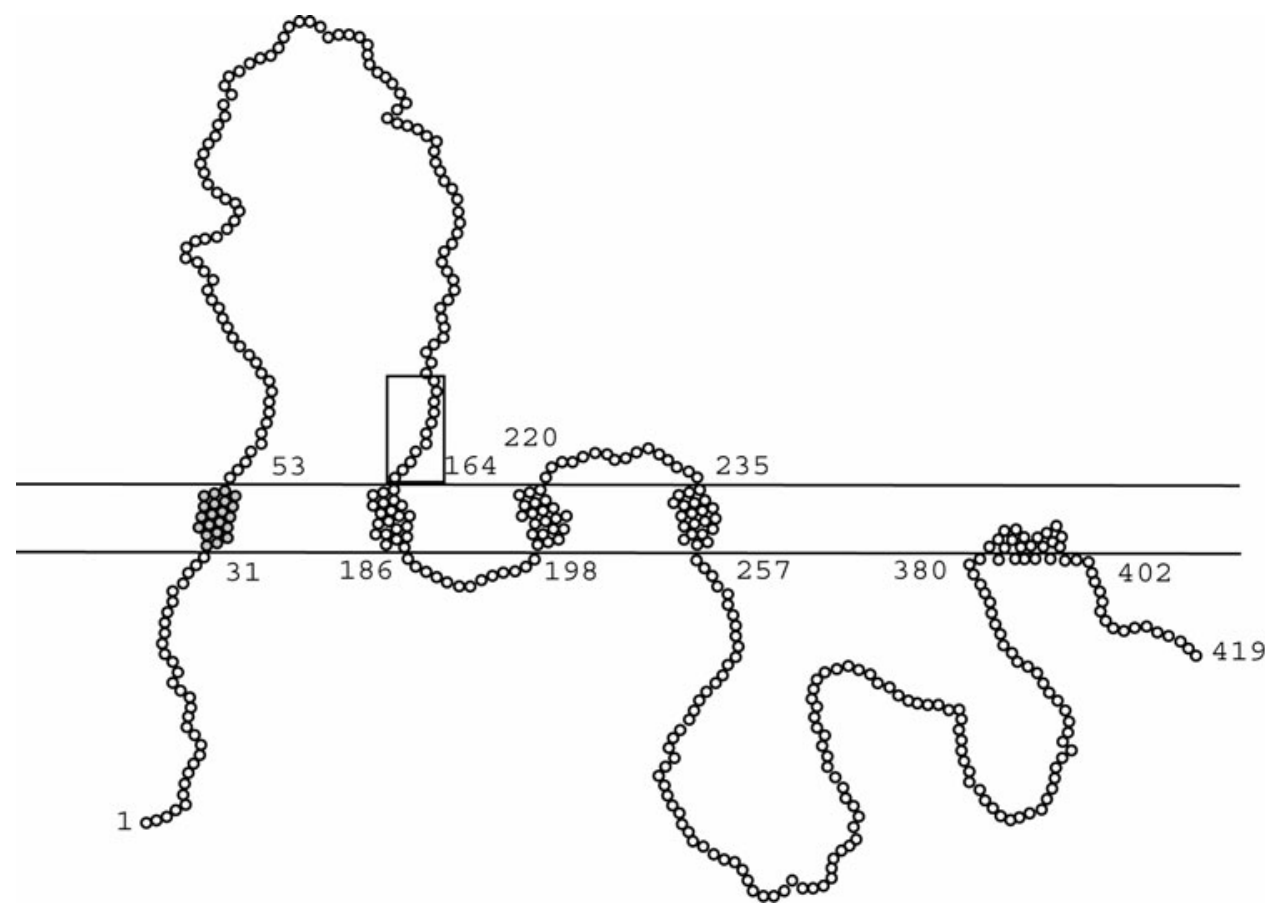

Fig. 6. SmTOR protein model according to data generated with TopPred and PSIPREDView program. Numbers indicated refer to the first and last amino acid of the corresponding intra- or extracellular domain. The peptide sequence shown to bind C2 is boxed. Alphahelical transmembrane domains 1-4 are at positions 32-52, 165-185, 199-219 and 236-256 respectively. Transmembrane domain one acting as signal anchor is shaded in grey. 
1

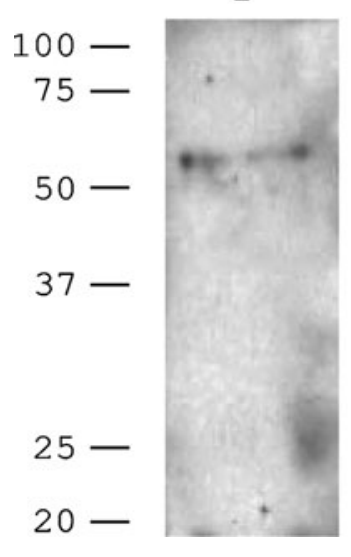

2

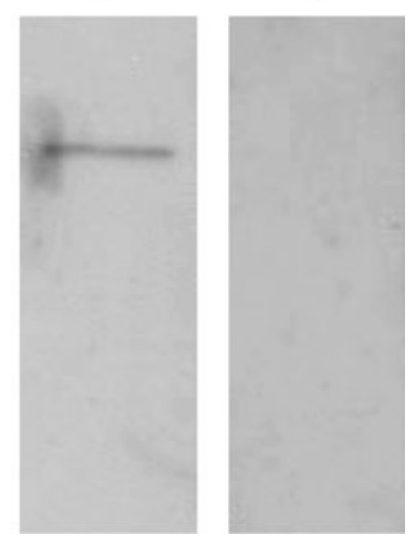

Fig. 7. Western blot of adult worm tegument preparation. Polyclonal anti-ed1, anti-ed2 antibody and pre-immune serum (lanes 1, 2 and 3 respectively).

infection. SmTOR might be essential at that specific time-point. Many complement proteins are produced by epidermal cells (Timar et al. 2007) and several authors have shown that complement proteins diffuse from the vascular compartment to the dermis and epidermis (Kool et al. 2007; Hansell et al. 2008). The complete complement cascade is active in the skin as indicated by the role of complement in many human blistering skin diseases (Lessey et al. 2008). SmTOR might at that time help the parasite to escape complement attack.

We had previously not been able to replicate the amplification of the short version ORF of the TOR receptor homologue from Schistosoma genomic DNA or from vertebrate genomic DNA of various sources (Inal et al. 2005b). This is now understandable since the gene has 3 large introns not described previously. In addition, we did not find the sequence or fragments of it when performing BLASTn searches on different vertebrate genomes. DeMarco et al. (2007) recently analysed the controversy about the origin of schistosome albumin, and demonstrated it to be of hamster origin. In his discussion, he suggested that CRIT might be an example of reverse contamination from the schistosome into the vertebrate samples. Our present data are in agreement with DeMarco's comments. We must now consider our previous observations as being due to contamination and non-specific antibody staining (Inal et al. 2005 a, b; Moll et al. 2006).

Previously, for both ShTOR and SmTOR, transcripts of $1.2 \mathrm{~kb}$ and $1.35 \mathrm{~kb}$ cloned from adult worm cDNA libraries had been reported to contain an open reading frame of $0.86 \mathrm{~kb}$, as proteins of 31.7 and $31 \cdot 2 \mathrm{kDa}$ were detected in adult worm preparations (Inal, 1999). Alignment of the region published as 5' UTR of ShTOR (Inal, 1999) with SmTOR exon 2 sequenced resulted in a nearly perfect match (alignment not shown). SjTOR cDNA and protein (Liu et al. 2006) are of the same length as we now found in
S. mansoni. We therefore suggest SmTOR to be longer than originally described with the former ATG lying within the second exon of the gene. The translation initiation site we suggest for SmTOR does not lie in a classical Kozak sequence (Kozak, 1984), but possible variation around the translation initiation codon has been reported for invertebrates (Nakagawa, 2008).

TOR protein had been shown on the surface of the adult worm by immunohistochemistry (Inal, 1999). We detected a band at approximately $55 \mathrm{kDa}$ in adult worm tegument membrane preparation using 2 different polyclonal antibodies generated against ed1 and ed 2 respectively. The SmTOR amino acid sequence contains a potential N-linked glycosylation site at position 138. The protein detected might be the glycosylated form, as its theoretical molecular weight was calculated as $46.7 \mathrm{kDa}$. SmTOR in alignment with $\mathrm{SjTOR}$ protein shows $76 \%$ identity and $84 \%$ similarity. TOR was found by Liu in S. japonicum in cercariae, schistosomula and adult worm (Liu et al. 2006), whereas no proteomic analysis of the schistosome tegument of adult worms identified peptides belonging to SmTOR (van Balkom et al. 2005 ; Braschi et al. 2006 b; Braschi and Wilson, 2006). This might be due to the low abundance of the protein in the tegument of the adult worm (Skelly and Wilson, 2006).

Based on the secondary structure analysis described above we generated a hypothetical protein model for SmTOR. SmTOR (or CRIT) was previously thought to span the membrane 3 times (hence the ' $\mathrm{T}$ ' for rispanning). The longer protein we found is very likely to be tetraspanning, as suggested also for $\mathrm{SjTOR}$, which allows us to suggest a change in the name of the molecule, but without a change in the abbreviations used. The amphiphatic helix inserted partially in the membrane is present in SjTOR and SmTOR, although there is high variability of amino acid sequence at this site between the two species.

SmTOR had been renamed to CRIT (complement $\mathrm{C} 2$ receptor inhibitor trispanning) according to its putative function to bind $\mathrm{C} 2$. The former N-terminal extracellular domain ed1 of ShTOR as isolated peptide had been shown to bind C2 and with its C-terminal 11 amino acids designated as H17 peptide being the active binding site (Inal and Schifferli, 2002). Incubation with this peptide has been shown to interfere with the binding of $\mathrm{C} 2$ to $\mathrm{C} 4 \mathrm{~b}$ and with $\mathrm{C} 2$ cleavage by complement $\mathrm{C} 1 \mathrm{~s}$, thus blocking complement activation by the classical pathway. The new tetraspanning SmTOR protein still contains the same amino acid sequence in the extracellular domain 1 known to bind $\mathrm{C} 2$. Whether this sequence binds $\mathrm{C} 2$ in vivo on schistosomes remains to be explored, and it is interesting to note several differences in it between $S$. mansoni and $S$. japonicum. It will be interesting to test whether these differences alter $\mathrm{C} 2$ binding to TOR/CRIT. 

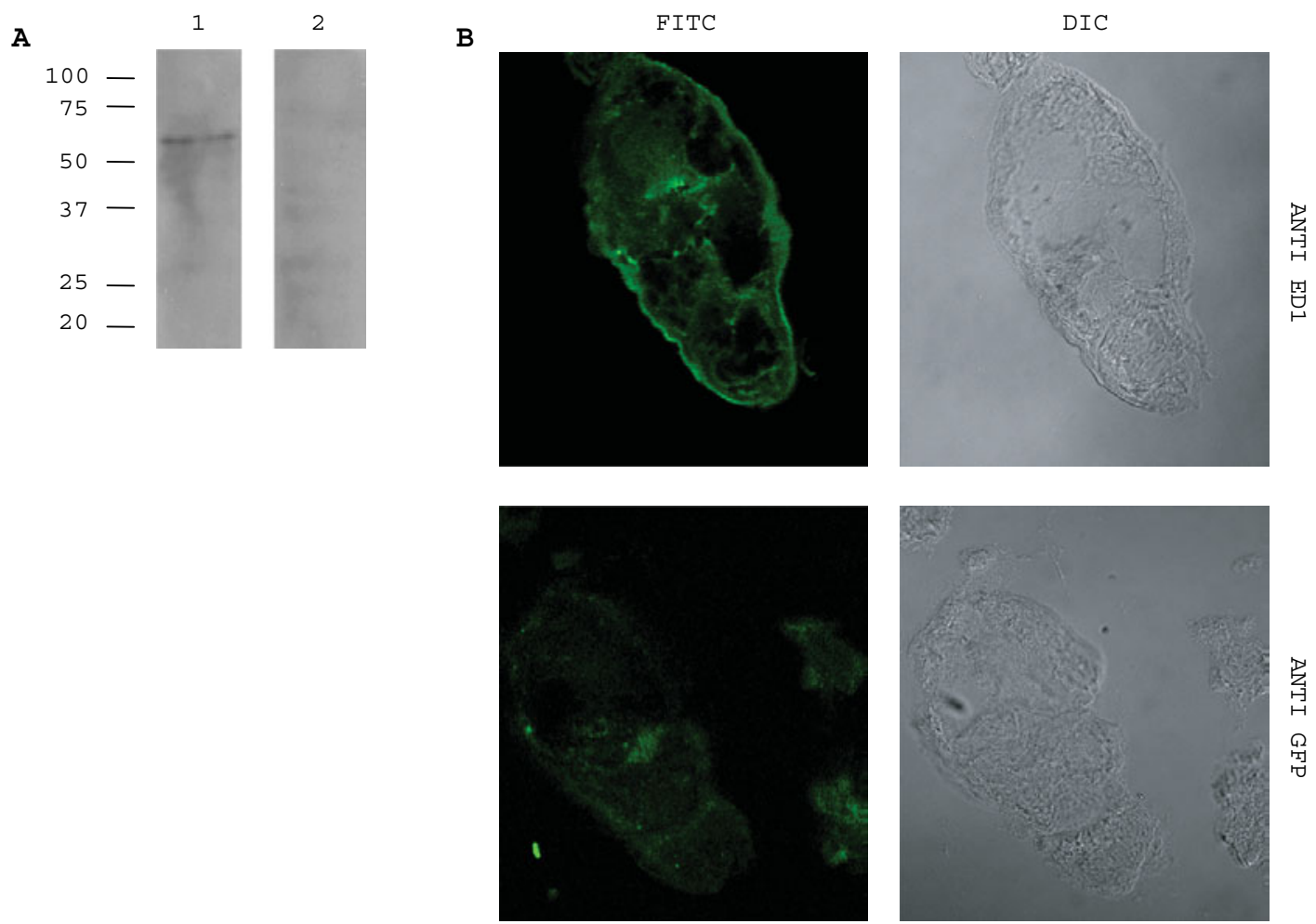

Fig. 8. SmTOR detection with a monoclonal anti-ed1 antibody AbyD04644.1. (A) Adult worm tegument preparation. Western blot probed with monoclonal anti-ed1 (lane 1) or anti-ed1 pre-incubated with 100-fold excess of ed1 peptide as control (lane 2). (B) Immunofluorescent labelling of cryosections through cercariae. Anti-ed1 labelled section top left panel and anti-GFP labelled control section bottom left panel.

\section{A}

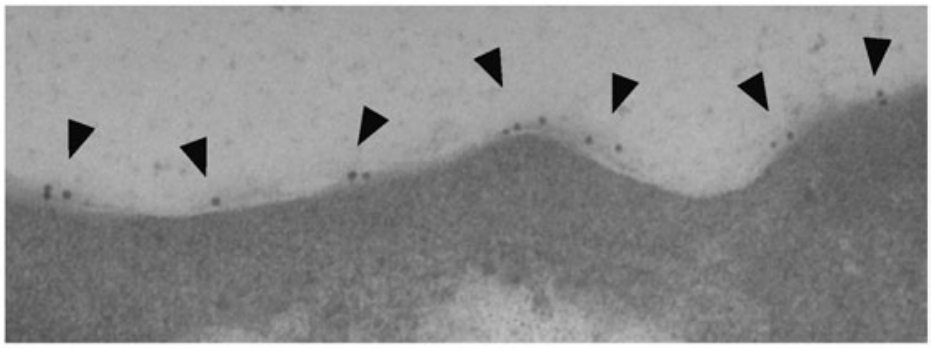

B

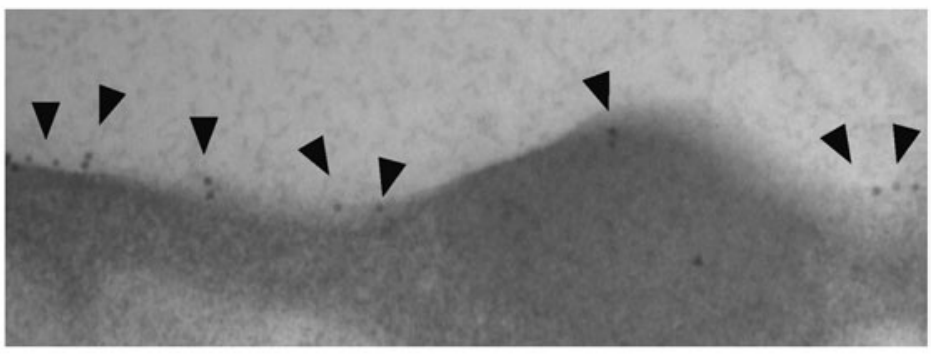

C

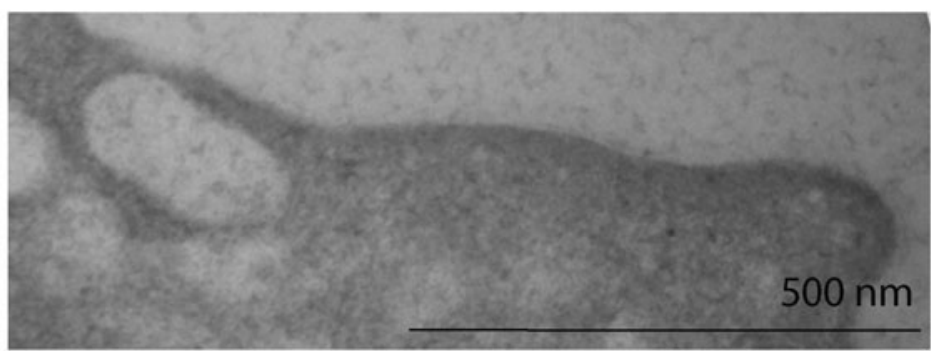

Fig. 9. Localization of SmTOR on tegument of cercariae using electron microscopy. Immunogold labelling is indicated by arrows. Sections stained with (A) polyclonal anti-ed1 antibody, (B) polyclonal anti-ed2 antibody and (C) pre-immune serum. Secondary antibody only was negative (not shown). 
We thank Dr J. Chollet, Y. Hagenmüller and C. List (Swiss Tropical Institute) for providing the Schistosoma material. Further thanks go to Vesna Olivieri and Ursula Sauder (Microscopy Center, Pharmacenter, University of Basel) for performing the electron microscopy, Brigitte Schneider and Beatrice Dolder (Infectious Diseases and Neurosurgery, University Hospital Basel) for helping with the cryosections and to the group of Professor A. Merlo (Neurosurgery, University Hospital Basel) for providing technical resources and support. Sequence data used to generate the genomic map were produced by the Wellcome Trust Sanger Institute and are available from www. genedb.org. This work was supported by the Swiss National Foundation (320000-116839).

\section{REFERENCES}

Bergquist, N. R., Leonardo, L. R. and Mitchell, G. F. (2005). Vaccine-linked chemotherapy: can schistosomiasis control benefit from an integrated approach? Trends in Parasitology 21, 112-117.

Braschi, S., Borges, W. C. and Wilson, R. A. (2006a). Proteomic analysis of the schistosome tegument and its surface membranes. Memorias Do Instituto Oswaldo Cruz 101, 205-212.

Braschi, S., Curwen, R. S., Ashton, P. D., Verjovski-Almeida, S. and Wilson, A. (2006b). The tegument surface membranes of the human blood parasite Schistosoma mansoni: a proteomic analysis after differential extraction. Proteomics 6, 1471-1482.

Braschi, S. and Wilson, R. A. (2006). Proteins exposed at the adult schistosome surface revealed by biotinylation. Molecular E Cellular Proteomics 5, 347-356. doi: 10.1074/mcp.M500287-MCP200

Brouwers, J., Skelly, P. J., Van Golde, L. M. G. and Tielens, A. G. M. (1999). Studies on phospholipid turnover argue against sloughing of tegumental membranes in adult Schistosoma mansoni. Parasitology 119, 287-294.

Claros, M. G. and Vonheijne, G. (1994). TopPredII - an improved software for membrane-protein structure predictions. Computer Applications in the Biosciences 10, 685-686.

Correa-Oliveira, R., Caldas, I. R. and Gazzinelli, G. (2000). Natural versus drug-induced resistance in Schistosoma mansoni infection. Parasitology Today 16, 397-399.

Correa-Oliveira, R., Pearce, E. J., Oliveira, G. C., Golgher, D. B., Katz, N., Bahia, L. G., Carvalho, O. S., Gazzinelli, G. and Sher, A. (1989). The human immune-response to defined immunogens of Schistosoma mansoni - elevated antibody levels to Paramyosin in stool-negative individuals from 2 endemic areas in Brazil. Transactions of the Royal Society of Tropical Medicine and Hygiene 83, 798-804.

Dalton, J. P., Day, S. R., Drew, A. C. and Brindley, P. J. (1997). A method for the isolation of schistosome eggs and miracidia free of contaminating host tissues. Parasitology 115, 29-32.

DeMarco, R., Mathieson, W., Dillon, G. P. and Wilson, R. A. (2007). Schistosome albumin is of host, not parasite, origin. International Fournal for Parasitology 37, 1201-1208. doi: 10.1016/j.ijpara. 2007.03.004
Hamburger, J., Xu, Y. X., Ramzy, R. M., Jourdane, J. and Ruppe1, A. (1998). Development and laboratory evaluation of a polymerase chain reaction for monitoring Schistosoma mansoni infestation of water. American Fournal of Tropical Medicine and Hygiene 59, 468-473.

Hansell, E., Braschi, S., Medzihradszky, K. F., Sajid, M., Debnath, M., Ingram, J., Lim, K. C. and McKerrow, J. H. (2008). Proteomic analysis of skin invasion by blood fluke larvae. PLoS Neglected Tropical Diseases 2, e262.

Hooker, C. W. and Brindley, P. J. (1996). Cloning and characterisation of strain-specific transcripts encoding triosephosphate isomerase, a candidate vaccine antigen from Schistosoma japonicum. Molecular and Biochemical Parasitology 82, 265-269.

Hui, K. M., Magnadottir, B., Schifferli, J. A. and Inal, J. M. (2006). CRIT peptide interacts with factor B and interferes with alternative pathway activation. Biochemical and Biophysical Research Communications 344, 308-314. doi: 10.1016/j.bbrc.2006.03.101

Hui, K. M., Orriss, G. L., Schirmer, T., Magnadottir, B., Schifferli, J. A. and Inal, J. M. (2005). Expression of functional recombinant von Willebrand factor-A domain from human complement $\mathrm{C} 2$ : a potential binding site for $\mathrm{C} 4$ and CRIT. The Biochemical Fournal 389, 863-868. doi : 10.1042/bj20050183

Inal, J. M. (1999). Schistosoma TOR (trispanning orphan receptor), a novel, antigenic surface receptor of the blood-dwelling, Schistosoma parasite. Biochimica et Biophysica Acta-Gene Structure and Expression 1445, 283-298.

Inal, J. M. (2005). Complement C2 receptor inhibitor trispanning: from man to schistosome. Springer Seminars in Immunopathology 27, 320-331. doi : 10.1007/s00281-005-0009-9

Inal, J. M., Hui, K. M., Miot, S., Lange, S., Ramirez, M. I., Schneider, B., Krueger, G. and Schifferli, J. A. (2005b). Complement C2 receptor inhibitor trispanning: A novel human complement inhibitory receptor. Fournal of Immunology 174, 356-366.

Inal, J., Miot, S. and Schifferli, J. A. (2005a). The complement inhibitor, CRIT, undergoes clathrindependent endocytosis. Experimental Cell Research 310, 54-65. doi: 10.1016/j.yexcr.2005.07.003

Inal, J. M. and Schifferli, J. A. (2001). C4 beta chain peptide interferes with the formation of the classical pathway C2 convertase. Molecular Immunology 38, 97.

Inal, J. M. and Schifferli, J. A. (2002). Complement C2 receptor inhibitor trispanning and the beta-chain of $\mathrm{C} 4$ share a binding site for complement C2. Fournal of Immunology 168, 5213-5221.

Inal, J. M. and Sim, R. B. (2000). A Schistosoma protein, Sh-TOR, is a novel inhibitor of complement which binds human C2. FEBS Letters 470, 131-134.

Jones, D. T. (1999). Protein secondary structure prediction based on position-specific scoring matrices. Fournal of Molecular Biology 292, 195-202.

Kemp, W. M., Brown, P. R., Merritt, S. C. and Miller, R. E. (1980). Tegument-associated antigen modulation by adult male Schistosoma mansoni. Fournal of Immunology 124, 806-811.

Kemp, W. M., Damian, R. T. and Greene, N. D. (1976). Immunocytochemical localisation of $\operatorname{IgG}$ on 
adult Schistosoma mansoni tegumental surfaces. Fournal of Parasitology 62, 830-832.

Kemp, W. M., Merritt, S. C. and Rosier, J. G. (1978). Schistosoma mansoni: Identification of immunoglobulins associated with tegument of adult parasites from mice. Experimental Parasitology 45, 81-87.

Klabunde, J., Berger, J., Jensenius, J. C., Klinkert, M. Q., Zelck, U. E., Kremsner, P. G. and Kun, J. F. J. (2000). Schistosoma mansoni: Adhesion of mannan-binding lectin to surface glycoproteins of cercariae and adult worms. Experimental Parasitology 95, 231-239.

Kool, J., Reubsaet, L., Wesseldijk, F., Maravilha, R. T., Pinkse, M. W., D’Santos, C. S., Van Hilten, J. J., Zijlstra, F. J. and Heck, A. J. (2007). Suction blister fluid as potential body fluid for biomarker proteins. Proteomics 7, 3638-3650.

Kozak, M. (1984). Compilation and analysis of sequences upstream from the translation start site in eukaryotic mRNAs. Nucleic Acids Research 12, 857-872.

Lessey, E., Li, N., Diaz, L. and Liu, Z. (2008). Complement and cutaneous autoimmune blistering diseases. Immunologic Research 41, 223-232.

Linder, E. and Huldt, G. (1983). Antibody-independent binding and activation of complement by Schistosoma mansoni adult worms. Parasite Immunology 5, 183-194.

Liu, F., Lu, J., Hu, W., Wang, S. Y., Cui, S. J., Chi, M., Yan, Q., Wang, X. R., Song, H. D., Xu, X. N., Wang, J. J., Zhang, X. L., Zhang, X., Wang, Z. Q., Xue, C. L., Brindley, P. J., McManus, D. P., Yang, P. Y., Feng, Z., Chen, Z. and Han, Z. G. (2006). New perspectives on host-parasite interplay by comparative transcriptomic and proteomic analyses of Schistosoma japonicum. Plos Pathogens 2, 268-281. doi: 10.1371/ journal.ppat.0020029

Livak, K. J. and Schmittgen, T. D. (2001). Analysis of relative gene expression data using real-time quantitative PCR and the 2(-Delta Delta C(T)) method. Methods 25, 402-408.

Marikovsky, M., Parizade, M., Arnon, R. and Fishelson, Z. (1990). Complement regulation on the surface of cultured schistosomula and adult worms of Schistosoma mansoni. European Fournal of Immunology 20, 221-227.

McGuffin, L. J., Bryson, K. and Jones, D. T. (2000). The PSIPRED protein structure prediction server. Bioinformatics 16, 404-405.

McManus, D. P. and Loukas, A. (2008). Current status of vaccines for schistosomiasis. Clinical Microbiology Reviezus 21, 225-242.

Minard, P., Dean, D. A., Jacobson, R. H., Vannier, W. E. and Murrell, K. D. (1978). Immunization of mice with Co-60 irradiated Schistosoma mansoni cercariae. American Fournal of Tropical Medicine and Hygiene 27, 76-86.

Moll, S., Lange, S., Mihatsch, M., Dragic, Z., Schifferli, J. and Inal, J. (2006). CRIT is expressed on podocytes in normal human kidney and upregulated in membranous nephropathy. Kidney International 69, 1961-1968.

Nakagawa, S. (2008). Diversity of preferred nucleotide sequences around the translation initiation codon in eukaryote genomes. Nucleic Acids Research 36, 861-871.
Pearce, E. J., Hall, B. F. and Sher, A. (1990). Host-specific evasion of the alternative complement pathway by schistosomes correlates with the presence of a phospholipase C-sensitive surface molecule resembling human decay accelerating factor. Fournal of Immunology 144, 2751-2756.

Ramalhopinto, F. J., Gazzinelli, G., Howells, R. E., Motasantos, T. A., Figueiredo, E. A. and Pellegrino, J. (1974). Schistosoma mansoni-defined system for stepwise transformation of cercaria to schistosomule in vitro. Experimental Parasitology 36, 360-372.

Rasmussen, K. R. and Kemp, W. M. (1987). Schistosoma mansoni: interactions of adult parasites with the complement system. Parasite Immunology $\mathbf{9}$, 235-248.

Roberts, S. M., MacGregor, A. N., Vojvodic, M., Wells, E., Crabtree, J. E. and Wilson, R. A. (1983). Tegument surface membranes of adult Schistosoma mansoni: development of a method for their isolation. Molecular and Biochemical Parasitology 9, 105-127.

Ross, A. G. P., Bartley, P. B., Sleigh, A. C., Olds, G. R., Li, Y. S., Williams, G. M. and McManus, D. P. (2002). Current concepts - schistosomiasis. New England Fournal of Medicine 346, 1212-1220.

Ruppel, A., McLaren, D. J., Diesfeld, H. J. and Rother, U. (1984). Schistosoma mansoni: escape from complement-mediated parasiticidal mechanisms following percutaneous primary infection. European Fournal of Immunology 14, 702-708.

Skelly, P. J. (2004). Immunoparasitology series: intravascular schistosomes and complement. Trends in Parasitology 20, 370-374. doi: 10.1016/j.pt.2004.05.007

Skelly, P. J. and Wilson, R. A. (2006). Making sense of the schistosome surface. Advances in Parasitology 63, 185-284. doi : 10.1016/s0065-308x(06)63003-0

Steinmann, P., Keiser, J., Bos, R., Tanner, M. and Utzinger, J. (2006). Schistosomiasis and water resources development: systematic review, meta-analysis, and estimates of people at risk. Lancet Infectious Diseases 6, 411-425.

Stek, M., Minard, P., Dean, D. A. and Hall, J. E. (1981). Immunization of baboons with Schistosoma mansoni cercariae attenuated by gamma irradiation. Science 212, 1518-1520.

Tendler, M. and Simpson, A. J. (2008). The biotechnology-value chain: development of Sm14 as a schistosomiasis vaccine. Acta Tropica 108, 263-266.

Timar, K. K., Dallos, A., Kiss, M., Husz, S., Bos, J. D. and Asghar, S. S. (2007). Expression of terminal complement components by human keratinocytes. Molecular Immunology 44, 2578-2586.

Tran, M. H., Pearson, M. S., Jeffrey, M. B., Smyth, D. J., Jones, M. K., Duke, M., Don, T. A., McManus, D. P., Correa-Oliveira, R. and Loukas, A. (2006). Tetraspanins on the surface of Schistosoma mansoni are protective antigens against schistosomiasis. Nature Medicine 12, 835-840.

Van Balkom, B. W. M., Van Gestel, R. A., Brouwers, J., Krijgsveld, J., Tielens, A. G. M., Heck, A. J. R. and Van Hellemond, J. J. (2005). Mass spectrometric analysis of the Schistosoma mansoni tegumental sub-proteome. Fournal of Proteome Research 4, 958-966. doi: $10.1021 / \mathrm{pr} 050036 \mathrm{w}$ 